Farm animal welfare - who writes the rules?

Occasional Publication No. 23 - British Society of Animal Science 1999

edited by A. J. F. Russel, C. A. Morgan, C. J. Savory, M. C. Appleby and T. L. J. Lawrence

\title{
Aggression in pigs: an example of using basic research in an applied context
}

\author{
H. W. Erhardt, M. Mendl ${ }^{1} \ddagger$, and D. D. Ashley ${ }^{2}$ \\ ${ }^{1}$ Scottish Agricultural College, Edinburgh, West Mains Road, Edinburgh EH9 3JG \\ ${ }^{2}$ Veterinary School, University of Glasgow, Glasgow G61 1QH
}

\section{Introduction}

Most animal welfare researchers agree that a mixture of applied and basic research is required to address the complex problems in this area. However, basic research is sometimes more difficult to justify, particularly in the current climate where the public, politicians and media often measure the importance of research primarily in terms of the economic benefits it can deliver. Basic research may thus be dismissed as pointless by the public at large. Here we provide an example of basic research in animal behaviour and welfare which, by addressing a fundamental question about behavioural organization in animals, has resulted in an applied research programme partly funded by commercial organizations, which may in the long-term have important economic and welfare consequences for the pig industry.

The study presented here was part of a 3-year project on the extent to which individual behavioural differences in pigs can be interpreted as characteristics of animal 'personality'. 'Do pigs have personality?' is a good example of a basic research project which at first sight might appear to have no applications. However, this research may in fact provide an important approach to tackling the welfare problems caused by high levels of aggression between pigs.

The aim of this project was to determine whether attack latency of individual pigs as recorded in a controlled test situation predicts elements of aggressive behaviour in a different situation, namely when groups of unfamiliar pigs were mixed. If this was the case, it was suggested that aggressiveness could be regarded as a personality trait.

Present addresses:

† Macaulay Land Use Research Institute, Craigiebuckler, Aberdeen AB15 8QH.

$\ddagger$ Department of Clinical Veterinary Science, University of Bristol, Bristol BS40 5DU.

\section{Material and methods}

The attack latency test

In this test, an intruder pig is introduced into the test pig's home pen, and the time from when the test pig first makes contact with the intruder to when it attacks is recorded. Immediately after an attack, the intruder is removed from the pens and returned to its own home pen. If no attack occurred within $3.5 \mathrm{~min}$ after introduction of the intruder, the test is terminated and an attack latency of 3.5 min assigned to the test pig. This test was carried out on 2 days consecutively with 85 and on 78 pigs in 2 years consecutively (age 11 weeks), and with 53 pigs 4 weeks apart (age 7 and 11 weeks).

The mixing

One hundered and fifteen pigs were categorized according to their average attack latency into high and low aggressive pigs ( $\mathrm{H}$ and $\mathrm{L} ; 20.6 \pm 2.9$ and $156.5 \pm 8.5 \mathrm{~s}$ respectively). Eighty-eight of these pigs were then mixed into new groups of eight pigs per group. Each new group contained four pigs from each of two litters. The new groups contained $4 \mathrm{H}+$ $4 \mathrm{H}$ (four groups), $4 \mathrm{H}+4 \mathrm{~L}$ (four groups) and $4 \mathrm{~L}+4 \mathrm{~L}$ (three groups). The groups were observed and their behaviour recorded on days $0,1,2,6$ and 7 after mixing. Behaviours recorded were number, duration and intensity of fights (as measured by the number of skin lesions on the pigs from the winner litter in each group) and their lying preference (whether pigs avoided lying down next to a pig from the unfamiliar litter or not). The lying preference score ranges from ' +1 ' (unfamiliar pigs were always avoided) to ' -1 ' (unfamiliar pigs were never avoided). A score of ' 0 ' indicated that both happened equally often.

\section{Results}

Attack latencies were consistent across time (Spearman rank order correlations between tests on consecutive days (two replicates): $r_{\mathrm{s}}=0.56$ (no. $=85$, $P<0.001$ ) and $r_{\mathrm{s}}=0.73$ (no. $=78, P<0.001$ ) and across 4 weeks: $r_{s}=0.57$ (no. $=53, P<0.001$; for more information see Erhard and Mendl, 1997). 
When the pigs were categorized into high and low aggression pigs ( $\mathrm{H}$ and $\mathrm{L}$ ) according to their attack latency, and mixed into new groups, we found that attack latency predicted the time spent fighting (time (s) spent fighting per pig in the $2 \mathrm{~h}$ post mixing: $\mathrm{H} /$ H: 443.6 , s.e. $168 \cdot 0 ; \mathrm{H} / \mathrm{L}: 82 \cdot 8$, s.e. $54 \cdot 5 ; \mathrm{L} / \mathrm{L}: 144 \cdot 0$, s.e. 7.75; ANOVA, $\left.F_{2,8}=3.12, P<0.10\right)$ as well as the intensity of fighting (number of skin lesions on pigs from winner litter: $\mathrm{H} / \mathrm{H}: 84.5$, s.e. $3.95 ; \mathrm{H} / \mathrm{L}: 20.5$, s.e. $5 \cdot 67$; L/L: $36 \cdot 1$, s.e. $10 \cdot 67$; ANOVA, $F_{2,8}=28 \cdot 27$, $P<0.001$ ) and the speed of group integration (lying preference score: $\mathrm{H} / \mathrm{H}: 0 \cdot 41$, s.e. $0 \cdot 12 ; t=3.44$, no. $=4$, $P<0.05$; H L L 0.45 , s.e. $0.12 ; t=3.70$, no. $=4, P<0.05$; L/L: 0.09 , s.e. $0 \cdot 16 ; t=0.53$, no. $=3, P>0.05$; for more detail see Erhard et al. 1997).

\section{Discussion}

The results show that attack latency was consistent across time and predictive of other elements of aggressive behaviour in a different situation (elements of fighting behaviour and group integration after mixing in a group environment). Aggressiveness may therefore be considered a personality trait in pigs.

These results have a value beyond the basic understanding of pig behaviour. Many studies which aimed at finding ways of reducing aggression after mixing, found considerable individual difference in the performance of aggressive behaviour (e.g. Kelley et al., 1980; McGlone and Morrow, 1988; Mount and Seabrook, 1993), which increases the number of animals needed to detect significant treatment differences. If the variation within treatment can be reduced by prior assessment of the individuals and by including this information in the experimental design, the number of individuals required to detect treatment differences can be reduced. Thus the number of pigs undergoing experimental mixing will be reduced, which is of importance, since mixing aggression poses serious welfare problems (e.g. Petherick and Blackshaw, 1987).

Erhard et al. (1997) reported that a major part of the problems occurring at mixing was due to the presence of pigs with short attack (SA) latencies. They fought more vigorously when mixed with other
SA pigs and performed more biting/chasing behaviour when mixed with long attack latency pigs. Groups containing SA pigs were also slower to integrate, which was reflected in their lying preference. We therefore suggested that it may be beneficial for the welfare of pigs, if the proportion of SA pigs in the population could be reduced (Erhard et al., 1997).

Information on individual aggressiveness in pigs may thus be used in breeding programmes to reduce the proportion of high aggressive pigs in the population and thereby the suffering and economic losses caused by aggression in the pig industry as a whole. This possibility is now the subject of research funded jointly by government, pig industry companies and animal welfare charities.

Thus, a project which appeared not to have any applications has resulted in information which can be applied to improve animal welfare, with consequent economic implications.

\section{Acknowledgements}

The initial study was funded by the BBSRC, UFAW and SOAEFD.

\section{References}

Erhard, H. W. and Mendl, M. 1997. Measuring aggressiveness in growing pigs in a resident-intruder situation. Applied Animal Behaviour Science 54: 123-136

Erhard, H. W., Mendl, M., and Ashley, D. D. 1997. Individual aggressiveness can be measured and used to reduce aggression after mixing. Applied Animal Behaviour Science 54: 137-151

Kelley, K. W., McGlone, J. J., and Gaskins, C. T. 1980. Porcine aggression: measurements and effects of crowding and fasting. Journal of Animal Science 50: 336-341

McGlone, J. J. and Morrow, J. 1988. Reduction of pig agonistic behavior by androstenone. Journal of Animal Science 66: $880-884$

Mount, N. C. and Seabrook, M. F. 1993. A study of aggression when group housed sows are mixed. Applied Animal Behaviour Science 36: 377-383

Petherick, J. C. and Blackshaw, J. K. 1987. A review of the factors influencing the aggressive and agonistic behaviour of the domestic pig. Australian Journal of Experimental Agriculture 27: 605-611 